N. Kallel, M. Timoumi (Preparatory Inst. Engineering Studies, Tunisia)

\title{
SUBHARMONICS OF A NONCONVEX NONCOERCIVE HAMILTONIAN SYSTEM
}

\section{СУБГАРМОНІКИ НЕОПУКЛОЇ НЕКОЕРЦИТИВНОЇ ГАМІЛЬТОНОВОЇ СИСТЕМИ}

In this paper we study the existence of multiple periodic solutions of the Hamiltonian system

$$
J \dot{x}+u^{*} \nabla G(t, u(x))=e(t),
$$

where $u$ is a linear map, $G$ is a $C^{1}$-function, and $e$ is a continuous function.

Досліджепо питапня про іспувашия кратних періодичиих розв'язків гамільтонової системи

$$
J \dot{x}+u^{*} \nabla G(t, u(x))=e(t),
$$

де $и$-лінійле віјюбражепия, $G-C^{1}$-фупкція та $e-$ пеперервиа фупкція.

1. Introduction. In this paper we are interested for the existence of periodic solution of the noncoercive Hamiltonian system

$$
J \dot{x}+u^{*} \nabla G(t, u(x))=e(t),
$$

where $u: \mathbb{R}^{2 n} \rightarrow \mathbb{R}^{m}, 1 \leq m \leq 2 n$, is a linear map not identically null with adjoint $u^{*} ; G: \mathbb{R} \times \mathbb{R}^{n} \rightarrow \mathbb{R},(t, y) \rightarrow G(t, y)$ is a continuous function, $T$-periodic in the first variable $(T>0)$, differentiable with respect to the second variable and its derivative $\nabla G$ is continuous; $e: \mathbb{R} \rightarrow \mathbb{R}^{2 n}$ is a continuous $T$-periodic function with mean value zero, and

$$
J=\left(\begin{array}{cc}
0 & -I_{n} \\
I_{n} & 0
\end{array}\right)
$$

is the standard symplectic matrix.

There are many papers studying the multiplicity of periodic solutions of the Hamiltonian system $\dot{x}=J H^{\prime}(t, x)$. Numerous results were obtained by using many different techniques, for example Morse theory, minimax methods, etc. However, most of the results concerning the existence of subharmonic solutions have used the convexity and coercivity assumptions on $H$, see [ $1-4]$.

Our first result is the following.

Theorem 1. Assume that $G$ satisfies:

$\left(G_{1}\right) \quad \exists M>0: \forall t \in \mathbb{R}, \forall x \in \mathbb{R}^{m} \quad\|\nabla G(t, x)\| \leq M$;

$\left(G_{2}\right)$ either

(i) $\lim _{|x| \rightarrow+\infty} G(t, x)=+\infty$, uniformly in $t \in[0, T]$,

or

(ii) $\lim _{|x| \rightarrow+\infty} G(t, x)=-\infty$, uniformly in $t \in[0, T]$,

then the Hamiltonian system

$$
J \dot{x}+u^{*} \nabla G(t, u(x))=e(t)
$$

has at least one $T$-periodic solution. 
In the case where the forcing term $e$ is null, we obtain the following theorem.

Theorem 2. Under the assumptions $\left(G_{1}\right),\left(G_{2}\right)$, the Hamiltonian system

$\left(\mathcal{H}_{e}\right)$ possesses for all integer $k \geq 1$ a $k T$-periodic solution $x_{k}$ satisfjing

$$
\lim _{k \rightarrow+\infty}\left\|x_{k}\right\|_{\infty}=+\infty,
$$

where $\|x\|_{\infty}=\sup \{|x(t)|, t \in \mathbb{R}\}$.

Concerning the minimality of the period, we have the following result.

Theorem 3. If the Hamiltonian $G$ satisfies $\left(G_{1}\right)$ and the assumption

$\left(G_{2}^{\prime}\right)$ either

(i') $\lim _{|x| \rightarrow+\infty}\langle\nabla G(t, x), x\rangle=+\infty$, uniformly in $t \in[0, T]$,

or

(ii') $\lim _{|x| \rightarrow+\infty}\langle\nabla G(t, x), x\rangle=-\infty$, uniformly' in $t \in[0, T]$,

then for any sufficiently large prime number $k$, the system $\left(\mathscr{H}_{0}\right)$ possesses a $k T$ periodic solution with minimal period $k T$.

2. Preliminaries [5]. Let $X=W \oplus Z$ be a Banach space and $X_{n}=W_{n} \oplus Z_{n}$ be a sequence of closed subspaces with $Z_{0} \subset Z_{1} \subset \ldots \subset Z, \quad W_{0} \subset W_{1} \subset \ldots \subset W, 1<$ $<\operatorname{dim} W_{n}<\infty$. For every $\varphi: X \rightarrow \mathbb{R}$ we denote by $\varphi_{n}$ the function $\varphi$ restricted to $X_{n}$. Let us recill that, for any $A \subset X, A_{n}=A \cap X_{n}$.

Definition 1. Let $c \in \mathbb{R}$ and $\varphi \in C^{1}(X, \mathbb{R})$. The functional $\varphi$ satisfies the $(P S)_{c}^{*}$ condition if every sequence $\left(x_{n_{j}}\right) \subset X$ satisfying: $\varphi_{n_{j}}\left(x_{n_{j}}\right) \rightarrow c, \quad \varphi_{n_{j}}^{\prime}\left(x_{n_{j}}\right) \rightarrow$ $\rightarrow 0$, possesses a subsequence which converges in $X$ to a critical point of $\varphi$.

Theorem 4 (Generalized saddle point theorem). Let $\varphi \in C^{\prime}(X, \mathbb{R})$. Assume that there exists $r>0$ such that, with $Y=\{w \in W ;|w|=r\}$ :

a) $\sup _{\gamma} \varphi \leq \inf _{Z} \varphi$;

b) $\varphi$ is bounded from above on $A=\{x \in W ;\|x\| \leq r\}$;

c) $\varphi$ satisfies the $(P S)_{c}^{*}$ condition, where $c=\inf _{A \in \mathcal{R}} \varphi \sup _{x \in A} \varphi(x)$, with

$$
\mathcal{A}=\left\{A \subset X ; A \text { is closed, } Y \subset A, \operatorname{cat}_{\chi, Y}^{\infty}(A)=1\right\} .
$$

Then $c$ is a critical value of $\varphi$ and $c \geq \inf _{Z} \varphi$.

Remark 1. In a) we may replace $Z$ by $q+Z, q \in W$.

3. Proof of Theorem 1. We will prove here the case where $G$ satisfics $\left(G_{2}\right)(\mathrm{i})$, the case $\left(G_{2}\right)$ (ii) is the same.

Before giving a variational formulation of $\left(\mathcal{H}_{e}\right)$, some preliminary materials on function spaces and norms are needed.

Let $L^{2}\left(S^{1}, \mathbb{R}^{2 n}\right)$ be the space of square integrable functions defined on $S^{1}=$ $=\mathbb{R} / T \mathbb{Z}$, with value in $\mathbb{R}^{2 n}$. Each function $x \in L^{2}\left(S^{1}, \mathbb{R}^{2 n}\right)$ has a Fourier expansion

$$
x(t)=\sum_{m \in \mathbb{Z}} \exp \left(\frac{2 \pi}{T} m t J\right) \hat{x}_{m},
$$

where $\hat{x}_{m} \in \mathbb{R}^{2 n}$ and $\sum_{m \in Z}\left|\hat{x}_{m}\right|^{2}<\infty$.

Set

$$
H^{1 / 2}\left(S^{1}, \mathbb{R}^{2 n}\right)=\left\{x \in L^{2}\left(S^{1}, \mathbb{R}^{2 n}\right) ;\|x\|_{H^{1 / 2}}<\infty\right\},
$$


where

$$
\|x\|_{H^{1 / 2}}=\left[\sum_{m \in \mathbf{Z}}(1+|m|)\left|\hat{x}_{m}\right|^{2}\right]^{1 / 2} .
$$

Consider the subspace

$$
X=\left\{x \in H^{1 / 2}\left(S^{\perp}, \mathbb{R}^{2 n}\right) ; x_{0} \in \operatorname{Ker} u^{\perp}\right\} .
$$

It is easy to check that the quadratic form $Q$ defined on $X$ by

$$
Q(x)=\frac{1}{2} \int_{0}^{T}\langle J \dot{x}, x\rangle d t
$$

satisfies, for a smooth $x \in X$

$$
Q(x)=-\pi \sum_{m \in Z} m\left|\hat{x}_{m}\right|^{2} .
$$

Set

$$
\begin{gathered}
X^{0}=(\operatorname{Ker} u)^{\perp}, \\
X^{+}=\left\{x \in X ; x(t)=\sum_{m \leq-1} \exp \left(\frac{2 \pi}{T} m t J\right) \hat{x}_{m} \text { a.e. }\right\}, \\
X^{-}=\left\{x \in X ; x(t)=\sum_{m \geq 1} \exp \left(\frac{2 \pi}{T} m t J\right) \hat{x}_{m} \text { a.e. }\right\},
\end{gathered}
$$

then $X=X^{+} \oplus X^{-} \oplus X^{0}$, and $X^{+}, X^{-}, X^{0}$ are respectively the subspaces of $X$ on wich $Q$ is positive definite, negative definite, snd null. $X^{+}, X^{-}, X^{0}$ are mutually orthogonal with respect to the associated inner product and mutually orthogonal in $L^{2}\left(S^{1}, \mathbb{R}^{2 n}\right)$.

These remarks show that if $x=x^{+}+x^{-}+x^{0} \in X$,

$$
\|x\|^{2}=\left|x^{0}\right|^{2}+Q\left(x^{+}\right)-Q\left(x^{-}\right),
$$

serves as an equivalent norm on $X$. Henceforth we use the norm defined in (2) as the norm for $X$. For cach $p \in] 1, \infty], X$ is compactly embedded in $L^{p}\left(S^{1}, \mathbb{R}^{2 n}\right)$. In particular there is $\alpha_{p}>0$ such that

$$
\|x\|_{L^{p}} \leq \alpha_{p}\|x\| \quad \forall x \in X .
$$

Now, consider the functional

$$
f(x)=\int_{0}^{T}\left[\frac{1}{2}\langle J \dot{x}, x\rangle+G(t, u(x))-\langle e(t), x\rangle\right] d t
$$

defined on the space $X$. The functional $f$ is continuously differentiable and verifies

$$
f^{\prime}(x) \cdot y=\int_{0}^{T}\left\langle J \dot{x}+u^{*} \nabla G(t, u(x))-e(t), y\right\rangle d t \quad \forall x, y \in X .
$$

We claim that the critical points of the functional $f$ correspond to the $T$-periodic solution of the system $\left(\mathcal{H}_{e}\right)$. Indeed, let $x$ be a critical point of $f$ on $X$, then by (4) there exists a constant $\xi \in \operatorname{Ker} u$ such that

$$
J \dot{x}+u^{*} \nabla G(t, u(x))-e(t)=\xi \quad \text { a. e. }
$$


Integrating (5) we obtain

$$
u^{*} \int_{0}^{T} \nabla G(t, u(x)) d t=T \xi
$$

so $\xi \in(\operatorname{Ker} u)^{\perp}$, which proves that $\xi=0$ and $x$ is a $T$-periodic solution of $\left(\mathcal{H}_{e}\right)$. Inversely, it is clear that every solution of $\left(\mathcal{H}_{e}\right)$ is a critical point of $f$ on $X$.

To find critical points of $f$ we shall apply the Generalized saddle point theorem to the functional $f$ on $X$ with these subspaces $W=X^{-}, Z=X^{+} \oplus X^{0}$ and the sequence of closed subspaces

$$
X_{n}=\left\{x \in X ; x(t)=\sum_{|m| \leq n} \exp \left(\frac{2 \pi}{T} m t J\right) \hat{x}_{m} \text { a.e. } \hat{x}_{0} \in(\operatorname{Ker} u)^{\perp}\right\} .
$$

- First, from (1), the assumption $\left(G_{1}\right)$ and the mean value theorem applied to the function $G\left(t_{,}\right)$, we have for $x=x^{0}+x^{+} \in Z$,

$$
f(x) \geq \frac{\pi}{2}\left\|x^{+}\right\|^{2}-c\left\|x^{+}\right\|+\int_{0}^{T} G\left(t, u\left(x^{0}\right)\right) d t,
$$

where $c$ is a constant. Since the function $u$ is invertible on $X^{0}$, we deduce from (6) and the assumption $\left(G_{2}\right)$ that $f(x)$ goes to infinity as $x$ goes to infinity in $Z$.

Second, there exist two constants $a, b \in \mathbb{R}$ such that for every $x \in W$

$$
f(x) \leq-\frac{\pi}{2}\|x\|^{2}+a\|x\|+b .
$$

Consequently there exists $r>0$ such that

$$
\sup _{Y} f \leq \inf _{Z} f,
$$

where $Y=\{x \in W ;\|x\|=r\}$. Furthermore, $f$ is bounded from above on $D_{r}$, where $D_{r}$ is the closed disk in $W$ centered in zero, with radius $r$.

Finally we will show that for all $c \in \mathbb{R}$, the functional $f$ satisfies the Palais Smale condition $(P S)_{c}^{*}$ with respect to $\left(X_{n}\right)$. Let $\left(x_{n_{j}}\right)$ be a sequence such that

$$
f\left(x_{n_{j}}\right) \rightarrow c, \quad f_{n_{j}}^{\prime}\left(x_{n_{j}}\right) \rightarrow 0, \quad n_{j} \rightarrow \infty, \quad x_{n_{j}} \in X_{n_{j}},
$$

we set $x_{n_{j}}=x_{n_{j}}^{0}+\tilde{x}_{n_{j}}$, where $x_{n_{j}}^{0}$ is the projection of $x_{n_{j}}$ onto $X^{0}$. We have from (1) and (4):

$$
\begin{gathered}
f_{n_{j}}^{\prime}\left(x_{n_{j}}\right)\left(x_{n_{j}}^{+}-x_{n_{j}}^{-}\right)=\pi \sum_{1 \leq|m| \leq n_{j}}|m|\left|\hat{x}_{j, m}\right|^{2}+ \\
+\int_{0}^{T}\left\langle u^{*} \nabla G(t, u(x))-e(t), x_{n_{j}}^{+}-x_{n_{j}}^{-}\right\rangle d t .
\end{gathered}
$$

It follows from (7) and the assumption $\left(G_{1}\right)$ that $\left(\tilde{x}_{n_{j}}\right)$ is bounded in $X$. Elsewhere, we have

$$
f\left(x_{n_{j}}\right)=\frac{1}{2} \int_{0}^{T}\left\langle J \tilde{x}_{n_{j}}, \tilde{x}_{n_{j}}\right\rangle d t+\int_{0}^{T} G\left(t, u\left(x_{n_{j}}\right)\right) d t-\int_{0}^{T}\left\langle e, \tilde{x}_{n_{j}}\right\rangle d t,
$$

so, by (7), the sequence $\left(\int_{0}^{T} G\left(t, u\left(x_{n_{j}}\right)\right) d t\right)$ is bounded. By applying the mean value theorem to the function $G(t,$.$) , there exist y_{n_{j}} \in X$ such that 


$$
\int_{0}^{T} G\left(t, u\left(x_{n_{j}}\right)\right) d t=\int_{0}^{T} G\left(t, u\left(x_{n_{j}}^{0}\right)\right) d t+\int_{0}^{T}\left\langle u^{*} \nabla G\left(t, u\left(y_{n_{j}}\right)\right), u\left(\tilde{x}_{n_{j}}\right)\right\rangle d t,
$$

and we deduce from the assumption $\left(G_{1}\right)$ that the sequence $\left(\int_{0}^{T} G\left(t, u\left(x_{n_{j}}^{0}\right)\right) d t\right)$ is bounded, so by the assumption $\left(G_{2}\right)(\mathrm{i}),\left(x_{n_{j}}^{0}\right)$ is also bounded in $X$. Up to a subsequence, we can assume that $x_{n_{j}} \rightarrow x$ and $x_{n_{j}}^{0} \rightarrow x^{0}$ in $X$. Note that

$$
\begin{gathered}
Q\left(x_{n_{j}}^{+}-x^{+}\right)=\left\langle f_{n_{j}}^{\prime}\left(x_{n_{j}}\right)-f^{\prime}(x), x_{n_{j}}^{+}-x^{+}\right\rangle- \\
-\int_{0}^{T}\left\langle\nabla G\left(t, u\left(x_{n_{j}}\right)\right)-\nabla G(t, u(x)), u\left(x_{n_{j}}^{+}-x^{+}\right)\right\rangle d t .
\end{gathered}
$$

This implies that $x_{n_{j}}^{+} \rightarrow x^{+}$in $X$. Similarly $x_{n_{j}}^{-} \rightarrow x^{-}$in $\mathrm{X}$. It follows then that $x_{n_{j}} \rightarrow x$ in $X$ and $f^{\prime}(x)=0$.

The function $f$ satisfies all the assumptions of the Generalized saddle point theorem, so $f$ has at least one critical point. The proof of Theorem 1 is completed.

4. Proof of Theorem 2. As in the proof of Theorem 1, we will prove the case where $G$ satisfies $\left(G_{1}\right)$ and $\left(G_{2}\right)(\mathrm{i})$, the case $\left(G_{2}\right)$ (ii) is the same. By making the change of variable $t \rightarrow t / k$, the system $\left(\mathcal{H}_{0}\right)$ transforms to

$$
J \dot{x}+k u^{*} \nabla G(k t, u(x))=0 .
$$

Hence to find $k T$-periodic solutions of $\left(\mathcal{H}_{0}\right)$, it suffices to find $T$-periodic solutions of $\left(\mathcal{H}_{k}\right)$, which are the critical points of the continuously differentiable functional

$$
f_{k}(x)=\frac{1}{2} \int_{0}^{T}\langle J \dot{x}, x\rangle d t+k \int_{0}^{T} G(k t, u(x)) d t
$$

defined on the space $X$ introduced above. By applying Theorem 4 to the functional $f_{k}$ on the space $X$, we prove as in Theorem 1 , that for all integer $k \geq 1$ the system $\left(\mathcal{H}_{k}\right)$ possesses a $T$-periodic solution $x_{k}$ such that

$$
f_{k}\left(x_{k}\right) \geq \inf _{x \in Z} f_{k}(x) \text {. }
$$

Now we will prove that the sequence $\left(x_{k}\right)$ obtained above has the following property

$$
\lim _{k \rightarrow \infty} \frac{1}{k} f_{k}\left(x_{k}\right)=\infty \text {. }
$$

This will be done by the following lemma.

Lemma 1. Assume that $G$ satisfies $\left(G_{2}\right)$, then:

$$
\lim _{k \rightarrow \infty} \inf _{x \in Z} \frac{f_{k}\left(k^{1 / 2}(\varphi+x)\right)}{k}=\infty,
$$

where $\varphi=\left(1 / \pi^{1 / 2}\right) \exp (2 \pi t J / T) e_{0}$ and $e_{0}$ is a paticular element in $X^{0}\left(e_{0} \neq 0\right)$.

Proof. Assume by contradiction that there exist sequences $k_{j} \rightarrow \infty, x_{j}=x_{j}^{+}+$ $+x_{j}^{0} \in Z$ and a constant $c \in \mathbb{R}$ such that

$$
f_{k_{j}}\left(k_{j}^{1 / 2}\left(\varphi+x_{j}\right)\right) \leq k_{j} c \quad \forall j \in \mathbb{N} .
$$

Taking $x_{j}=x_{j}^{+}+x_{j}^{0}, x_{j}^{i} \in X^{i}, i=+; 0$, by an easy calculation, we obtain 


$$
f_{k_{j}}\left(k_{j}^{1 / 2}\left(\varphi+x_{j}\right)\right)=k_{j}\left[\left\|x_{j}^{+}\right\|^{2}-\left\|e_{0}\right\|^{2}+\int_{0}^{T} G\left(k_{j} t, u\left(k_{j}^{1 / 2}\left(\varphi+x_{j}\right)\right)\right) d t\right],
$$

so by $\left(G_{2}\right)$ there exists a constant $\dot{c}_{1}>0$ such that

$$
f_{k_{j}}\left(k_{j}^{1 / 2}\left(\varphi+x_{j}\right)\right) \geq k_{j}\left(\left\|x_{j}^{+}\right\|^{2}-c_{1}\right)
$$

and we conclude, from inequality (11), that $\left(x_{j}^{+}\right)$is a bounded sequence in $X$. Taking a subsequence if necessary, we find $x^{+} \in X^{+}$such that

$$
x_{j}^{+}(t) \rightarrow x^{+}(t) \text { as } j \rightarrow \infty \text { for a. e. } t \in[0, T] .
$$

We claim that $\left(x_{j}^{0}\right)$ is also bounded in $X^{0}$. If we suppose otherwise, we easily deduce from (13) and the fact that $u$ is invertible on $X^{0}$ that

$$
\left|u\left(k_{j}^{1 / 2}\left(\varphi(t)+x_{j}^{+}(t)+x_{j}^{0}\right)\right)\right| \rightarrow \infty \text { as } j \rightarrow \infty \text { for a. e. } t \in[0, T] .
$$

Consequently by $\left(G_{2}\right)$ and Fatou's lemma, we obtain

$$
\int_{0}^{T} G\left(k_{j} t, u\left(k_{j}^{1 / 2}\left(\varphi(t)+x_{j}^{+}(t)+x_{j}^{0}\right)\right)\right) d t \rightarrow \infty \text { as } j \rightarrow \infty,
$$

and we deduce from (12) that

$$
\frac{f_{k_{j}}\left(k_{j}^{1 / 2}\left(\varphi+x_{j}^{+}+x_{j}^{0}\right)\right)}{k_{j}} \rightarrow \infty \text { as } j \rightarrow \infty,
$$

which contradicts (11) and proves our claim. Going if necessary to a subsequence, we can assume that there exists $x^{0} \in X^{0}$ such that $\varphi(t)+x_{j}^{+}(t)+x_{j}^{0} \rightarrow x(t)=\varphi(t)+x^{+}(t)+x^{0}$ as $j \rightarrow \infty$ for a. e. $t \in[0, T]$. By Fourier analysis, we have $x(t) \notin \operatorname{Ker} u$ for almost every $t \in[0, T]$. Therefore

$$
\left|u\left(k_{j}^{1 / 2}\left(\varphi(t)+x_{j}^{+}(t)+x_{j}^{0}\right)\right)\right| \rightarrow \infty \text { as } j \rightarrow \infty,
$$

and by $\left(G_{2}\right)$ and Fatou's lemma, we obtain (15), which contradicts (11). Thus (10) must hold. The proof of Lemma 1 is completed.

Hence Theorem 4 (Remark 1) implies that for all $k \in \mathbb{N}$, we have

$$
f_{k}\left(x_{k}\right)=b_{k} \geq \inf _{x \in Z} f_{k}\left(k^{1 / 2}(\varphi+x)\right),
$$

so, we have by Lemma 1 :

$$
\frac{b_{k}}{k} \rightarrow \infty \text { as } k \rightarrow \infty \text {. }
$$

We claim that $\left\|x_{k}\right\|_{\infty} \rightarrow \infty$ as $k \rightarrow \infty$. Indeed, if we suppose otherwise, $\left(x_{k}\right)$ possesses a bounded subsequence $\left(x_{k p}\right)$. Since

$$
\frac{f_{k}\left(x_{k}\right)}{k}=-\frac{1}{2} \int_{0}^{T}\left\langle u^{*} \nabla G\left(k t, u\left(x_{k}\right)\right), x_{k}\right\rangle d t+k \int_{0}^{T} G\left(k t, u\left(x_{k}\right)\right) d t
$$

the sequence $\left(b_{k_{p}} / k_{p}\right)$ is bounded, contrary to (9). Consequently, we have

$$
\lim _{k \rightarrow \infty}\left\|x_{k}\right\|_{\infty} \rightarrow \infty
$$


which implies that $v_{k}(t)=x_{k}(t / k)$, is a $k T$-periodic solution of the system $\left(\mathcal{H}_{0}\right)$ and verifies:

$$
\lim _{k \rightarrow \infty}\left\|v_{k}\right\|_{\infty}=\lim _{k \rightarrow \infty}\left\|x_{k}\right\|_{\infty}=\infty .
$$

That concludes the proof of Theorem 2 .

5. Proof of Theorem 3 . We begin the proof by the following lemma.

Lemma 2. Thee assumptions $\left(G_{1}\right),\left(G_{2}^{\prime}\right)$ imply the assumption $\left(G_{2}\right)$.

Proof. Assume for example $\left(G_{1}\right)$ and $\left(G_{2}^{\prime}\right)\left(\mathrm{i}^{\prime}\right)$ hold and let us prove $\left(G_{2}\right)(\mathrm{i})$. Let $R>0$ be such that

$$
|x| \geq R \Rightarrow\langle\nabla G(t, x), x\rangle \geq 1 \quad \forall t \in \mathbb{R} .
$$

Then, for $|x| \geq R$, we have

$$
\begin{gathered}
G(t, x)=G(t, 0)+\int_{0}^{R /|x|}\langle\nabla G(t, s x), x\rangle d s+\int_{R / x \mid}^{1}\langle\nabla G(t, s x), x\rangle d s \geq \\
\geq G(t, 0)-M R+\int_{R /|x|}^{1} \frac{d s}{s} \geq \inf _{t \in \mathbb{R}} G(t, 0)-M R+\log \left(\frac{|x|}{R}\right)
\end{gathered}
$$

and $\left(G_{2}\right)$ (i) follows.

Let $k$ be an integer $\geq 1$, we consider the functional $\varphi_{k}$

$$
\varphi_{k}(x)=\frac{1}{2} \int_{0}^{k T}\langle J \dot{x}, x\rangle d t+\int_{0}^{k T} G(t, u(x)) d t
$$

defined on the space

$$
X^{k}=\left\{x \in H^{1 / 2}\left(S_{k}^{!}, \mathbb{R}^{2 n}\right) ; x_{0} \in(\operatorname{Ker} u)^{\perp}\right\},
$$

where $S_{k}^{1}=\mathbb{R} / k T \mathbb{Z}$.

It is clear that $x \in X$ is a critical point of $f_{k}$ if and only if $v(t)=x(t / k)$ belongs to $X^{k}$ and is a critical point of $\varphi_{k}$. By Lemma 2, the assumptions $\left(G_{1}\right),\left(G_{2}\right)$ are satisfied, so we use Theorem 2.

Let $\left(v_{k}\right) \subset X^{k}$ be the sequence of critical points of $\varphi_{k}$ associated to the sequence $\left(x_{k}\right) \subset X$ of critical points of $f_{k}$ obtained in the proof of Theorem 2, the property (9) is written

$$
\lim _{k \rightarrow \infty} \frac{1}{k} \varphi_{k}\left(v_{k}\right)=\infty .
$$

On the other hand, let us denote by $S_{T}$ the set of $T$-periodic solutions of $\left(\mathcal{H}_{0}\right)$ which are in $X$. We claim that $S_{T}$ is bounded in $X$. Indeed, assume by contradiction that there exists a sequence $\left(v_{n}\right)$ in $S_{T}$ such that $\left\|v_{n}\right\|_{\infty} \rightarrow \infty$. Let us write $\tilde{v}_{n}=$ $=v_{n}^{+}+v_{n}^{-}$and $\bar{v}_{n}$ the mean value of $v_{n}$, then $v_{n}=\bar{v}_{n}+\bar{v}_{n}$. Multiplying both sides of the identity

$$
J \dot{v}_{n}+u^{*} \nabla G\left(t, u\left(v_{n}\right)\right)=0
$$

by $v_{n}^{+}-v_{n}^{-}$and integrating, we obtain

$$
\left\|\tilde{v}_{n}\right\|^{2}+\int_{0}^{T}\left\langle\nabla G\left(t, u\left(v_{n}(t)\right)\right), u\left(v_{n}^{+}-v_{n}^{-}\right)\right\rangle d t=0 .
$$


Using assumption $\left(G_{1}\right)$, we easily deduce that $\left(\tilde{v}_{n}\right)$ is bounded in $X$. So $\left(\vec{v}_{n}\right)$ is bounded in $L^{\infty}([0, T])$, and then $\left(u\left(\vec{v}_{n}\right)\right)$ is bounded in $L^{\infty}([0, T])$. The identity

shows then that

$$
\left\|v_{n}\right\|^{2}=\left|\bar{v}_{n}\right|^{2}+\left\|\tilde{v}_{n}\right\|^{2}
$$

$$
\lim _{n \rightarrow \infty}\left|\bar{v}_{n}\right|=\infty,
$$

and then

$$
\lim _{n \rightarrow \infty}\left|u\left(\bar{v}_{n}\right)\right|=\infty .
$$

Consequently, we obtain

$$
\lim _{n \rightarrow \infty} \min _{t \in[0 . T]}\left|u\left(v_{n}(t)\right)\right|=\infty .
$$

Multiplying (17) by $v_{n}$ and integrating, we get

$$
\int_{0}^{T}\left\langle J \dot{v}_{n}, v_{n}\right\rangle d t+\int_{0}^{T}\left\langle\nabla G\left(t, u\left(v_{n}\right)\right), u\left(v_{n}\right)\right\rangle d t=0 .
$$

Now, since $\left(\tilde{v}_{n}\right)$ is bounded in $X$, we deduce from (19) that

$$
\left(\int_{0}^{T}\left\langle\nabla G\left(t, u\left(v_{n}\right)\right), u\left(v_{n}\right)\right\rangle d t\right)
$$

is bounded.

But this is in contradiction with (18) and $\left(G_{2}^{\prime}\right)$. The claim follows immediately. As a consequence, $\varphi_{1}\left(S_{T}\right)$ is bounded, and, since for any $v \in S_{T}$ one has $\varphi_{k}(u)=$ $=k \varphi_{1}(u)$, we have

$$
\exists c>0: \quad \forall v \in S_{T}, \quad \forall k \geq 1 \quad \frac{1}{k}\left|\varphi_{k}(v)\right| \leq c .
$$

Consequently, (16) and (20) show that, for $k$ sufficiently large, $v_{k} \notin S_{T}$, and if $k$ is chosen to be a prime number, the minimal period of $v_{k}$ has to be $k T$ and the proof is completed.

1. Conley C., Zehnder E. Subharmonic solutions and Morse theory // Phisica A. - 1984, - 124. P. $649-658$.

2. Ekeland I., Hofer H. Subharmonics for convex nonautonomous Hamiltonian systems // Communs Pure and Appl. Math. - 1987, - 40, - P. 1 - 36 .

3. Rabinowitz P. H. On subharmonic solutions of Hamiltonian systems // Ibid. - 1980. - 33. P. $609-633$.

4. Timoumi $M$. Subharmonics of convex noncoercive Hamiltonian systems // Coll. Math. - 1992. Fasc.1. - P. $63-69$.

5. Fournier G., Timoumi M., Willem $M$. The limiting case for strongly indefinite functionals // Top. Meth. in Nonlinear Anal. - 1993. - 1. - P. 203 - 209. 